\title{
El manejo de los residuos sólidos y la actividad turística en Chetumal, México: una relación compleja
}

\section{Armando Alberto León-López}

Doctorando en Desarrollo Sostenible, División de Desarrollo Sustentable de la Universidad de Quintana Roo, Unidad Académica Cozumel. Área de interés: evaluación de políticas públicas socioambientales, sistemas complejos y educación para el desarrollo sostenible. Actualmente se desempeña como docente de asignaturas relacionadas con la generación del conocimiento científico en el nivel de educación superior.

armando.leonlpz@gmail.com

\section{Alfonso González Damián}

Doctor en Ciencias Sociales y Políticas por la Universidad Iberoamericana. Investigador nacional (SNI nivel 1) con las líneas de gestión socialmente sostenible de empresas y destinos turísticos y construcción social de la experiencia turística. Profesor investigador de la División de Desarrollo Sustentable de la Universidad de Quintana Roo, Unidad Académica Cozumel. gonzalezd@uqroo.edu.mx

\section{Crucita Aurora Ken}

Doctora en Ciencias del Desarrollo Regional por la Universidad Michoacana de San Nicolás de Hidalgo. Profesora del Departamento Económico-Administrativo de la Universidad de Quintana Roo. Ha publicado artículos sobre desarrollo regional, local y endógeno, gestión pública, turismo y relaciones económicas internacionales.

cruken@uqroo.edu.mx

\section{Inocente Bojórquez Báez}

Doctor en Ciencias Técnicas por el Instituto Superior Politécnico José Antonio Echeverría (Cuba). Investigador en el área de la construcción y la arquitectura tropical. Ha sido profesor de asignaturas como Resistencia de Materiales, Ecotecnologías, Heliodiseño y Dibujo en la División de Ciencias e Ingeniería de la Universidad de Quintana Roo. (pensionado)

inocente.bojorquez@gmail.com 


\section{El manejo de los residuos sólidos y la actividad turística en Chetumal, México: una relación compleja}

\section{Resumen}

En este análisis se relacionan los aspectos contextuales del turismo para develar el estilo de manejo de residuos sólidos en Chetumal. A través del estudio de caso, se articuló la conceptualización teórica del proceso del manejo de residuos sólidos en el enfoque de complejidad. Se abordaron, como escalas, los aspectos contextuales del destino turístico, los elementos para el flujo de residuos sólidos y el papel de los agentes sociales involucrados en la gestión. Para ello, se revisaron fuentes periódicas: diarios locales, informes técnicos y gubernamentales, que, junto con entrevistas complementarias a residentes, dieron cuenta de la evolución y el estilo de manejo. Se concluye que la inclusión de la contextualización turística y de zona fronteriza de la ciudad, junto a una nueva cultura de gobernanza, son importantes para el diseño de políticas sostenibles en el destino turístico.

\section{Palabras clave}

Complejidad; turismo; gobernanza; competitividad ambiental; residuos sólidos.

\section{Solid waste management and tourist activity in Chetumal, Mexico: a complex relationship}

\begin{abstract}
This research relates the contextual aspects of tourism to reveal the style of the solid waste management in Chetumal. Though case study approach, we articulate the theoretical conceptualization of the solid waste management process in the complexity approach. Contextual aspects of the tourist destination, elements of the solid waste flow and the role of the social actors involved in management were addressed as complex scales. For this, periodic sources were reviewed: local newspapers, technical and government reports, which, together with some interviews to residents reported on the evolution and management style. We conclude that the inclusion of tourist contextualization and border area of the city, together with a new culture of governance, are important for the design of sustainable policies in the tourist destination.
\end{abstract}

\section{Key words}

Complexity; tourism; governance; environmental competitiveness; solid waste.

\section{Gestão de resíduos sólidos e atividade turística em Chetumal, México: uma relação complexa}

\section{Resumo}

Nesta análise, os aspectos contextuais do turismo estão relacionados para revelar o estilo de gerenciamento de resíduos sólidos em Chetumal. Por meio do estudo de caso, a conceituação teórica do processo de gerenciamento de resíduos sólidos foi articulada na abordagem da complexidade. Os aspectos contextuais do destino turístico, os elementos para o fluxo de resíduos sólidos e o papel dos agentes sociais envolvidos na gestão foram abordados como escalas. Para isso, foram revisadas fontes periódicas: jornais locais, relatórios técnicos e governamentais, que, juntamente com entrevistas complementares aos moradores, deram conta da evolução e do estilo de gestão. Conclui-se que a inclusão da contextualização turística e da área de fronteira da cidade, juntamente com uma nova cultura de governança, são importantes para o desenho de políticas sustentáveis no destino turístico.

\section{Palavras chave}

Complexidade; turismo; governança, competitividade ambiental, resíduos sólidos. 


\section{El manejo de los residuos sólidos y la actividad turística \\ en Chetumal, México: una relación compleja}

\section{Introducción}

El turismo ha sido visto como un detonador económico para países en vías de desarrollo; sin embargo, también es sabido que trae consigo una serie de inconvenientes socioambientales aunados a la presión externa ejercida por los visitantes. Un ejemplo de las consecuencias de la actividad turística es la generación de residuos sólidos (RS) en el destino turístico (DT), por lo que colocar esta problemática en la agenda turística conllevaría reflexiones respecto de una mayor competitividad ambiental turística (Pulido-Fernández y Pulido-Fernández, 2015).

La adicionalidad dada por el turismo a una zona fronteriza recae en el fomento de la actividad empresarial, divisas, inversiones extranjeras, creación de empleo local e ingresos públicos, así como mejorar el desarrollo cultural y la conservación de los recursos naturales del DT (Orgaz y Moral, 2016). Sin embargo, el reto está en cómo conseguir permear dichos beneficios y reducir sus consecuencias. De esta forma, la gobernanza ha sido propuesta como herramienta para lograr el equilibrio sostenible, y se define como la interacción entre múltiples agentes sociales involucrados en la gestión turística, lo cual puede ser analizado en el enfoque de complejidad a través del estudio de los sistemas complejos (BAGGIo, ScotT Y COPPER, 2010).

La política pública internacional vigente para la gestión de residuos sólidos (GRS) es la llamada gestión integral, la cual es definida como un sistema en el que interactúan tres elementos principales: el papel de los agentes sociales involucrados, los elementos para el flujo de los RS y los aspectos externos — que fungen como contexto social, económico y ambiental- (Abarca-Moreno, MaAs y Hogland, 2015), lo cual habla de la complejidad funcional-estructural como política pública. Por otro lado, la interacción entre estos elementos podría impactar positivamente en el desempeño de la gestión, lo cual abonaría hacia la búsqueda de la sostenibilidad (Geissdoerfer; Savaget; Bocken y Hultink, 2016).

Siendo Chetumal un lugar con potencial turístico por su situación fronteriza, comercial y política, históricamente se ha enfrentado a una GRS inconstante, lo que se refleja en tiraderos clandestinos a cielo abierto tanto en la ciudad como en sus alrededores, inclusive en la bahía de Chetumal. Esto, aunado a una inadecuada disposición de los RS en el relleno sanitario, conlleva el riesgo de contaminación por lixiviados al manto freático (MANDujANo, 2002) y vulnera la sostenibilidad del patrimonio natural. 
1. Para efectos de la presente investigación, cuando se hable de gestión se hará referencia al conjunto de acciones normativas, operativas, financieras, de planeación, administrativas, sociales, educativas, de monitoreo, supervisión y evaluación para el manejo de RS. Asimismo, el manejo será visto como las actividades realizadas por los agentes a fin de lograr la gestión integral, tal como puede interpretarse en la Ley para la Prevención y Gestión Integral de los Residuos (LGPGIR, 2018).
Abordar la GRS en un DT en el enfoque de complejidad contribuiría a la visualización estructural de la problemática. En el trabajo, se relacionan los aspectos contextuales y de escalas espacio-temporales con los procesos evolutivos de la gestión a través de los puntos de vista político y ciudadano. Lo anterior, con la finalidad de develar el estilo de manejo ${ }^{1}$ de RS que se ha dado desde la fundación de la ciudad a finales del S. XIX, hasta su situación actual como zona fronteriza en lo que va del S. XXI.

Además de la visualización de la problemática en la particularidad territorial de la ciudad en cuestión, el estudio de las escalas en complejidad permite dar cuenta de algunas consideraciones metodológicas para futuras investigaciones interesadas en este abordaje teórico. Por último, como uno de los principales hallazgos se tiene que, en la ciudad, se da cuenta de la necesidad de una nueva cultura de gobernanza participativa para el diseño de políticas públicas inclusivas, consolidadas y sostenibles.

\subsection{Aproximación teórica}

El trabajo se enmarca en el enfoque de complejidad, en el cual se propone el abordaje de los sistemas complejos como recortes de la realidad, que fue señalada en la obra de Edgar Morin como compleja, abierta, evolutiva, no-lineal y caracterizada por estructuras que propician la emergencia de nuevas propiedades y elementos (VIGURI, 2019). En un replanteamiento del modelo llamado SISTUR (Sistema de Turismo), construido a partir de la Teoría General de Sistemas (Beni y Moesh, 2017; cp. Gullarducci y Fratucci, 2020), se señala la importancia de considerar las interacciones entre los elementos del sistema, tanto externos (ecosistema) como internos (red de agentes), y la emergencia de fenómenos accionados por agentes que recrean el sistema turístico en una rotación espacio-temporal dada y en un territorio específico. En otras palabras, se orientó dicho modelo hacia el enfoque de complejidad.

Por otro lado, los fenómenos emergentes y complejos pueden ser percibidos por los agentes sociales que los viven como aspectos positivos - como el incremento de la calidad de vida de los anfitriones- o como externalidades negativas, o también llamados retos para la sostenibilidad (Pulido-Fernández y Pulido-Fernández, 2015, p. 1316). Esta problemática es atendida por la GRS, que es vista como el conjunto de acciones para la toma de decisiones, y es resultado de la interacción de diversos agentes sociales involucrados —o red de agentes como sistema complejo-, que juntos materializan algún estilo de gestión o manejo, 
sean estos sostenibles o no, y que responde a su contexto por medio de la evolución de las políticas adoptadas por etapas sociohistóricas (JIMÉNEZ, 2015).

Esta red de agentes y sus interacciones pueden ser abordadas como mecanismos que se relacionan y coinciden en el formato de gobernanza en el DT, lo que promueve la emergencia de una nueva cultura local y reglas de colaboración hacia la sensibilización social para el tratamiento de los fenómenos socioambientales (NAva y ValenZuela, 2014), que, vistos como sistemas complejos y en contextos determinados, pueden detonar la gestión turística sostenible del territorio (GeissDoerfer et ÁL., 2016). Autores como GeRSHENSON (2013) sugieren abordar la complejidad a través de sus escalas temporales, de lugar y funcionales. Sobre esta lógica, se invoca la propuesta de ЈАсому (1992), quien sugiere que el análisis de las técnicas debe darse en tres dimensiones: la descripción de un panorama, indispensable para la comprensión sociohistórica; el objeto, que, para fines de este trabajo, es propiamente el manejo dado a los RS en el DT, y además, tomar en cuenta la figura ejecutora o sujeto, como la base pragmática en la toma de decisiones que explican la dinámica estructural-funcional (ver figura 1).

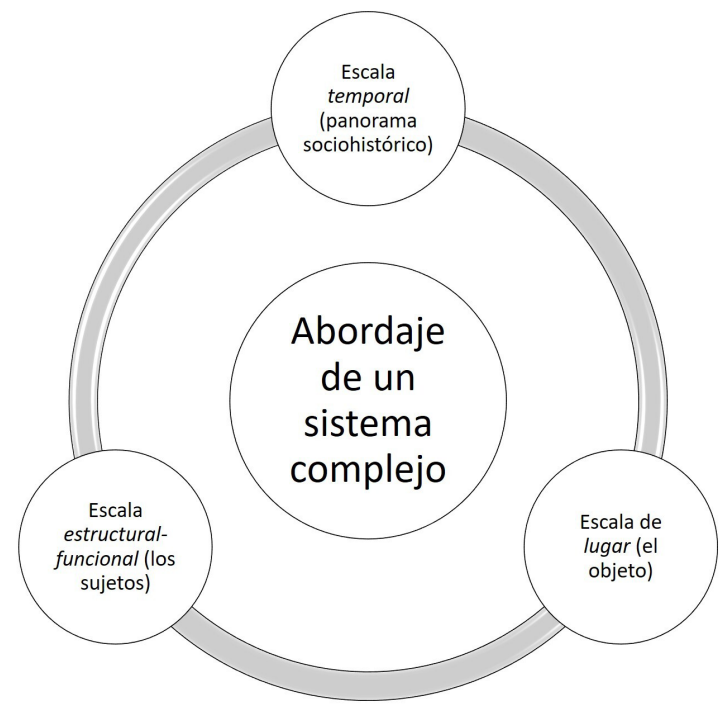

Figura 1. Escalas para el abordaje del sistema complejo para su estudio. Fuente: elaboración de los autores sobre la base de las propuestas de ЈасомY, 1992 y GERSHENSON, 2013 
Figura 2. Modelo sistémico de gestión integral de residuos

Fuente: de los autores con base a las reflexiones y propuestas de IsSOWAMA,

(2009 C. P. ABARCA-

MORENO ET AL., 2015)

$y$ VAN DE KLUNDERT Y ANSCHUTZ, (2001 C. P. JIMÉNEZ, 2017).
$\mathrm{Al}$ integrar las dimensiones de JACOMY (1992) en coincidencia con la propuesta de GERSHENSON (2013), se posibilita el estudio de los sistemas complejos, como es el caso de la GRS. De esta forma, al retomarse las categorías sugeridas en ABARCA-GueRRERo et Ál. (2015), junto con el concepto de gestión integral de RS para la sostenibilidad de VAN DE KLUNDERT y ANsChUTZ (2001, citado en JimÉnez, 2017), el abordaje queda en el estudio de las interacciones complejas y sostenibles a través de los aspectos externos de la GRS (contexto); los elementos para el flujo de los RS y el papel de los agentes sociales involucrados (ver figura 2).

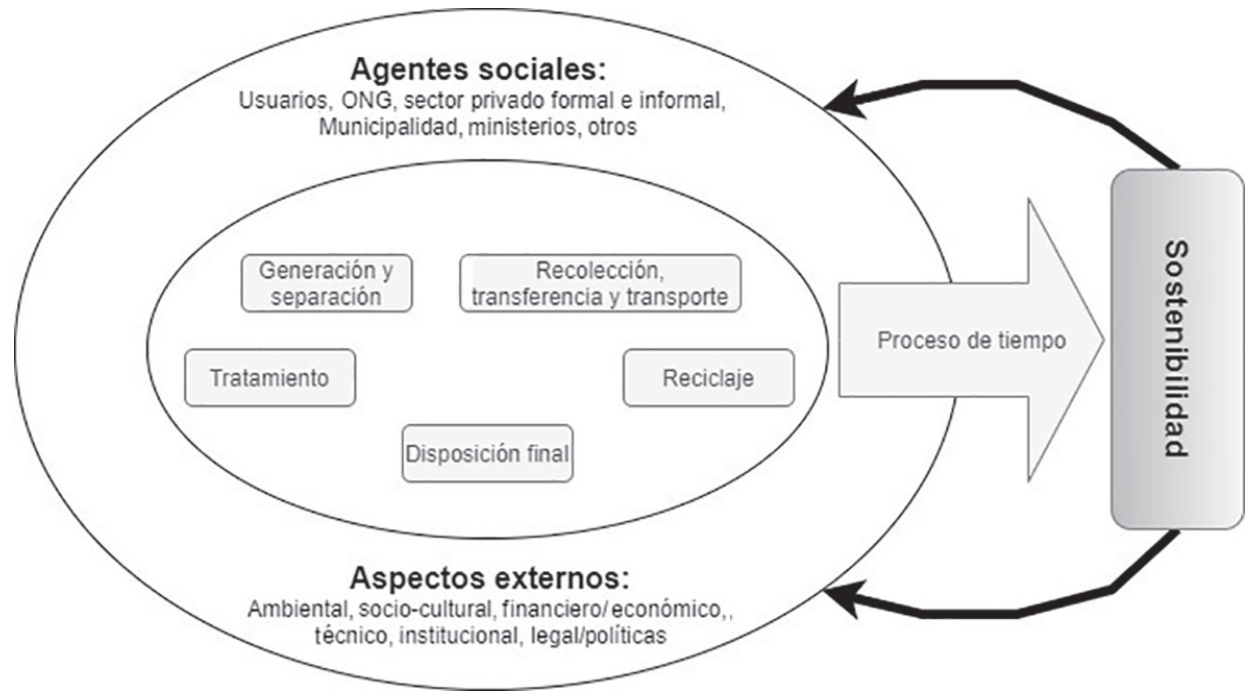

Esto se complementa con el posicionamiento del trabajo acerca de la sostenibilidad, que es entendida como el estado integral en equilibrio intra e intergeneracional de los aspectos sociales, económicos y ambientales de un sistema complejo. Además, dicho sistema se encuentra conformado por una red de agentes que son los responsables de decidir qué se debería de desarrollar y sostener, por cuánto tiempo y en beneficio de quién, a partir del contexto de un territorial dado (GEISSDOERFER et ÁL., 2016). 


\section{El manejo de los residuos sólidos y la actividad turística \\ en Chetumal, México: una relación compleja}

\subsection{Presentación del estudio de caso y aspectos contextuales: escala del lugar}

Chetumal, siendo cabecera del municipio de Othón P. Blanco y capital del estado de Quintana Roo, se ubica en el borde interior de la bahía de Chetumal, muy cercana a la frontera con Belice (ver figura 3). En la década de los 70 fue la ciudad con mayor número de habitantes en el estado, y tuvo una importante actividad comercial desde su decreto como "zona libre" en 1972 y hasta el año 1994 con la entrada en vigor del Tratado de Libre Comercio de América del Norte (TLCAN) (MacíAs, 2004).

Actualmente, con 154.243 habitantes (INEGI, 2010), la ciudad sigue dictando la política estatal como capital y haciéndose un lugar en la actividad turística en Quintana Roo, ya que tan solo en 2017 recibió a 495.976 visitantes, hecho que la colocó en el tercer puesto con respecto a la afluencia total en el estado, solo por debajo de destinos como la Riviera Maya y Cancún-Puerto Morelos, de acuerdo con la Secretaría de Turismo de Quintana Roo (SEDETUR, 2018).

Si bien, por un lado, esta afluencia puede ser traducida como gasto y derrame económico por parte de la actividad turística, por otro, hace reconocer una carga adicional a la gestión ambiental del DT. Por esta razón, uno de los retos urbanos relacionados es la GRS, la cual ha presentado una evolución inconstante e inadecuada, que pone en riesgo la salud pública y ambiental debido a la percolación de lixiviados en el sitio de disposición final, mal llamado relleno sanitario² (MANDUJANo, 2002).

A la problemática, se adiciona la presencia de vidrios de color, PET (politereftalato de etileno, un tipo de plástico), telas, pañales, desechables y unicel, que convierten a la bahía de Chetumal en un sitio clandestino para recepción de RS, debido principalmente a actividades recreativas locales y del turismo (Gamboa y CARriLlo, 2016). Asimismo, se ha reportado la presencia de residuos que arriban a la Costa Maya y la confirmación de microplásticos que tienen origen sea por el arrastre de corrientes marinas (GARCíA y OROPEZA, 2015) o por cruceros que transitan sobre el mar Caribe (Guevara; Flores; Canul; Aburto y Romero, 2011).

La situación pareciera no corresponder a los avances en materia jurídica y administrativa relacionados con la problemática ambiental en el país, pues la GRS se encuentra alineada a los estándares internacionales de la Agenda 21, garantizando el derecho a un medio ambiente por la Constitución Política Mexicana (Poder Ejecutivo, 2020), que permea a través de leyes nacionales, estatales, reglamentos y demás normatividad mexicana. Sin embargo, a nivel local es
2. Sitio que, a pesar de contar con infraestructura de relleno sanitario desde su implementación, opera como tiradero a cielo abierto y afecta la calidad de vida de comunidades y colonias aledañas (GUEVARA Y FLORES, 2011). 
urgente la actualización del reglamento de limpia, que debería ser vinculante a la instrumentación jurídica y administrativa que vela por el desarrollo sostenible (LEÓN-LóPEZ, 2016, p. 59).

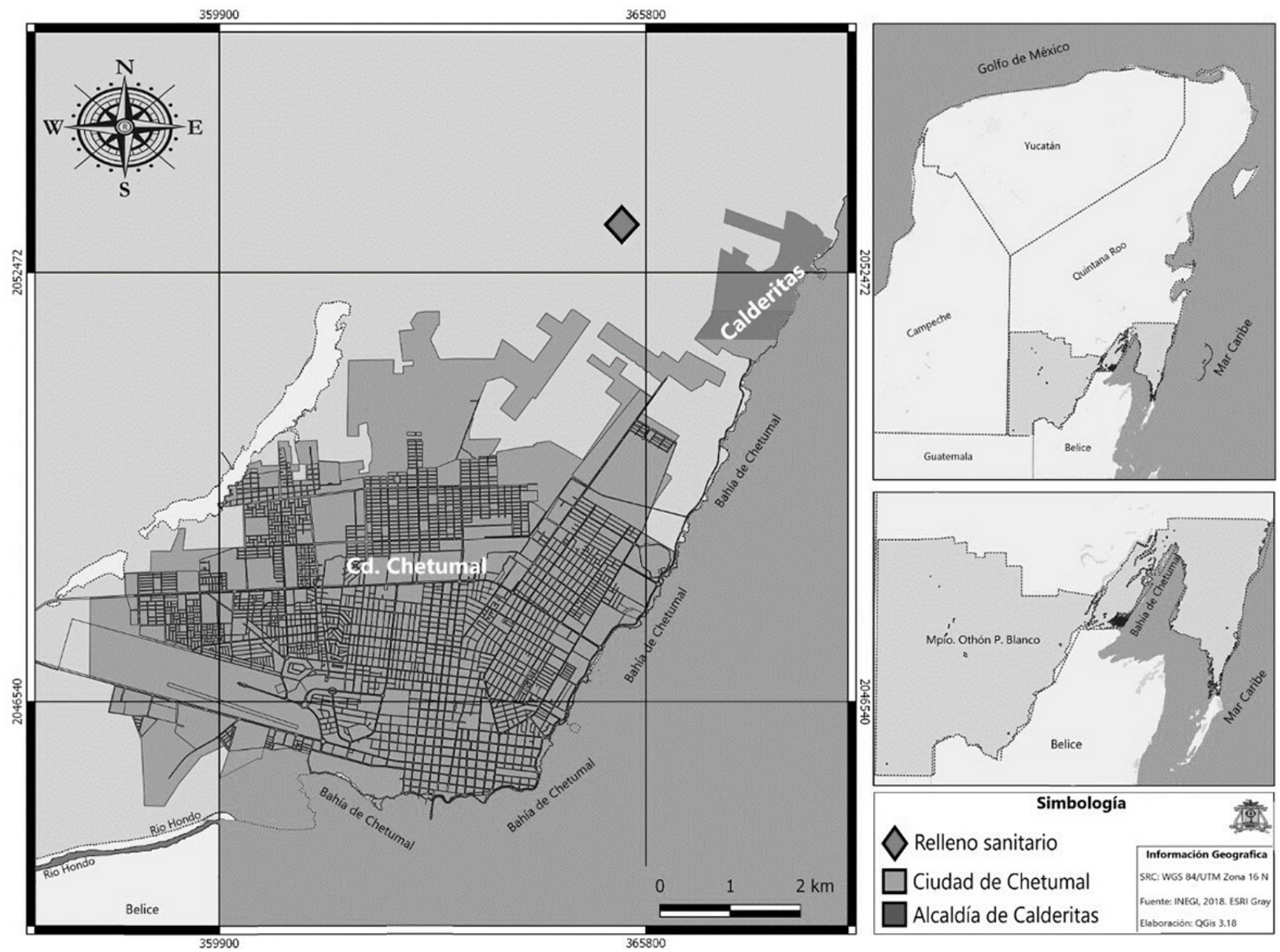

Figura 3. Mapa de ubicación de Chetumal y relleno sanitario. Fuente: elaboración por los autores 


\section{Metodología}

La investigación se diseñó como estudio de caso en tres fases, cada una enfocada en un aspecto del sistema complejo: (1) los aspectos externos de la GRS (contexto); (2) los elementos para el flujo de los RS y (3) el papel de los agentes sociales involucrados. En la primera fase, se realizó una revisión contextual del estudio de caso acerca de su vocación turística, territorio fronterizo y situación en cuanto a RS. Esta fase sirvió para la redacción de la sección de presentación del estudio de caso (sección 1.2.).

La segunda fase se apoyó en el análisis de la evolución del manejo de los RS en la ciudad. Para ello se consultaron fuentes periódicas, como diarios locales, informes técnicos e informes de gobiernos estatales y municipales del Archivo Histórico del estado de Quintana Roo, así como instancias gubernamentales, como la Secretaría de Turismo del Estado de Quintana Roo (SEDETUR, 2018). Se logró reconstruir el proceso relacionado con el manejo de los RS, desde el momento de fundación de la ciudad, hasta su dinámica fronteriza actual con el país de Belice (sección 3).

Como parte complementaria a la segunda fase, se incluyó la percepción de ciudadanos residentes que decidieran proporcionar su opinión en cuanto al servicio público de RS. Esta información fue recabada en diciembre de 2015, a través de dos preguntas abiertas que forman parte de la aplicación de un instrumento para una investigación previa en LEóN-López (2016); esta información fue procesada a través de la técnica de análisis del discurso (sección 3).

Para la tercera fase de la investigación (sección 4), se reflexionó acerca de la estructura funcional de la GRS en el DT (figura 2), con base en las categorías y reflexiones de análisis las sugeridas en ABARCA-GuerRero et AL. (2015) y JimÉNez (2017), pero tratadas como escalas de complejidad, lo cual se aborda en detalle en la aproximación teórica (sección 1.1).

\section{Evolución del manejo de residuos sólidos y las actividades turísticas en la ciudad de Chetumal: escala temporal}

\subsection{Datos históricos de un territorio con potencial de colonización}

A finales del S. XIX, el gobierno mexicano promovió la idea de crear un centro de población en las orillas de la bahía de Chetumal. Se fundó Payo Obispo — hoy Chetumal—en el 
año 1898, con la misión de cuartel para el control de los mayas en constante rebeldía y establecimiento de una aduana para el control de la invasión extranjera a la zona, promovida por la corona inglesa, para la explotación maderable (VARGAS, 2014).

A principios de los 1900, la descripción del sitio no solo aludía a una riqueza impresionante en flora y fauna, sino también se relataban cuestiones deplorables en lo sanitario, como la situación de las basuras (sic), la cual exigía el pronto alejamiento de estas hacia tiraderos lo más lejanos posible de la población y resolver la acumulación considerable en los toneles de hoteles, que podían pasar hasta tres meses sin ser vaciados (Secretaría de Agricultura y Fomento, 1918). Para 1967 —diez años después del paso del huracán Janet-, los informes de gobierno aún reflejaban la preocupación higienista para los 58.000 habitantes que conformaban lo que hoy es la capital del estado (Gobierno del TerRitorio de Quintana Roo, 1967).

\subsection{La problemática de limpieza y ornato de la ciudad turística}

Desde su fundación a principios del siglo XX como Territorio Federal, el estado de Quintana Roo encontró inserción en el comercio internacional a través de la producción primaria de chicle, copra y maderas preciosas. En los años 70 se registró un giro en la economía en torno al turismo, promovido principalmente por el programa de modernización en el norte del estado con la consolidación de Cancún como el principal centro turístico de masas de la región (Ken, 2014). La manera en que esto impactó en Chetumal en el ámbito ambiental fue que la gestión de las basuras se comenzó a percibir como una problemática de aspecto de limpieza y ornato para la ciudad, debido al interés de las autoridades de posicionarla como un lugar turístico, con base en el decreto de la "zona libre de Chetumal" en el año 1972.

Por aquella época, el Ayuntamiento apostaba a la participación ciudadana a través de campañas de limpieza, sin obtener mucha convocatoria. Para el año 1975, los agentes políticos de la época referían a la obligación moral de los chetumaleños para contribuir a la limpieza. En 1976, periódicos locales anunciaban como parte de la solución al problema la adquisición de un camión recolector para servir a las casas-habitación, establecimientos comerciales y edificios públicos. Se advertían sanciones a quienes sacaran antes la basura de sus hogares, para evitar malos olores y fauna nociva (NovEDADES DE QuiNTANA Roo, 1975).

Solo un año después, los medios de circulación celebraban la adquisición de tres máquinas barredoras para el servicio de limpieza de la población, junto al apoyo de 115 trabajadores en 
el servicio de limpieza (Ayuntamiento de Othón P. Blanco, s. f. a), que resultaban insuficientes de acuerdo con la percepción social de la época, por lo que el gobierno municipal, sin mucho éxito en la convocatoria, seguía exhortando a la población en la participación de la campaña permanente de limpieza (Ecos DeL CARIBE, 1976).

En el rubro de Aseo Urbano y Salud Pública, en el período 1983-1984, se llevaba a cabo la recolección de las basuras a través de dieciséis rutas de camiones recolectores, las mismas que incluían a las poblaciones aledañas de Calderitas y Subteniente López. Los camiones recolectores tipo cilindro eran tres y se apoyaban por unos siete volquetes (ver figura 4) para la recolección de 54.8 t/d de desperdicios y un costo de operación de $\$ 35.736 .000,00$ (treinta y cinco millones setecientos treinta y seis mil pesos mexicanos de aquella época) (Ayuntamiento de Othón P. Blanco, s. f. b).

Los trabajos de limpieza eran llevados a cabo con una plantilla de 92 obreros y dos supervisores. Las tareas realizadas eran: el barrido de cerca de 8719 m, $1287 \mathrm{~m}$ de raspado en orillas de banquetas y guarniciones y 104 de chapeo con un costo de $\$ 19.338 .000,00$ (diecinueve millones trescientos treinta y ocho mil pesos de la época con cero centavos). Sin embargo, en esos años, la especulación de la ocupación por la densificación de la población repercutió en la adquisición de terrenos que posteriormente se dejaban como lotes baldíos, que resultaban en potenciales basureros clandestinos (Ayuntamiento De Othón P. Blanco, s. f. b).

Con la adopción del programa Agenda 21 para la promoción de la sostenibilidad (Bustos y CHAcón, 2009), se explica el impulso tomado a la presentación de diversos tipos de proyectos ingenieriles y económicos. En el primer informe del gobierno de 1990-1993 se menciona la realización de dos grandes campañas de descacharrización y limpieza, con apoyo de veinticinco organizaciones federales, estatales y municipales, 1000 ciudadanos, 100 vehículos de apoyo y la recolección de 200 toneladas (t) de basura (Ayuntamiento de Othón P. Blanco, 1991).

En el segundo informe de la administración municipal de los años 1990-1993, el discurso incluía la participación de organizaciones de los sectores público, social y privado. Se contaba entonces con el apoyo de instituciones educativas, gubernamentales nacionales y locales, así como de militares para la campaña de limpieza. Para el año 1991 se reportaba la recolección a través de veinte rutas domésticas y seis comerciales, en atención de todas las colonias de Chetumal (Ayuntamiento de Othón P. Blanco, s. f. c). 
Figura 4. Equipos para el servicio de limpia y recolección de basura en el periodo de gobierno municipal 1981-1984
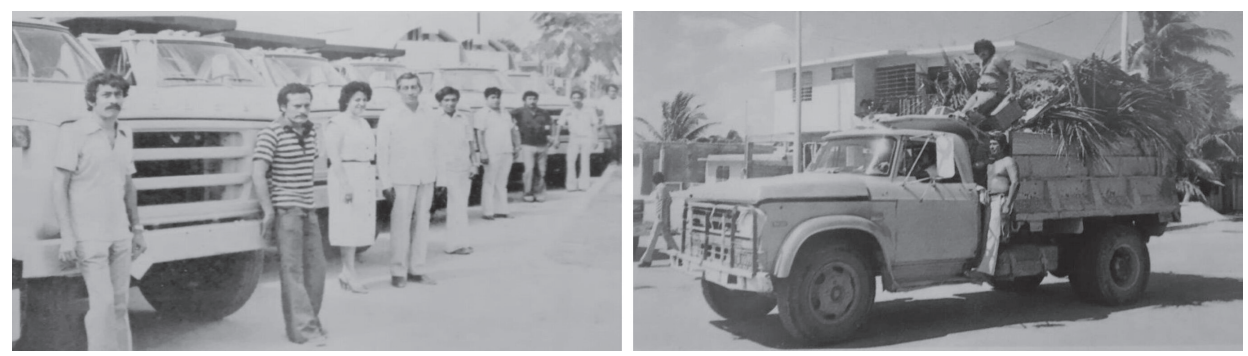

En el tercer informe del año 1993 se registró un incremento de la generación de basura en un $44 \%$ con respecto al año 1991, con 145.000 t recolectadas, a través de 26 rutas. Además, se hablaba de esfuerzos interinstitucionales coordinados entre la Subcomisión Técnica de la Comisión Municipal de Saneamiento Ambiental y Ecología, integrada en 1990 por el Centro de Investigaciones de Quintana Roo, la onceava Zona Naval Militar, la Secretaría de Salud, la Comisión Nacional del Agua y el Ayuntamiento, para el desarrollo de la edificación del Relleno Sanitario de la ciudad (Ayuntamiento de Othón P. Blanco, s. f. d).

El costo total de dicha obra se reportó de 11.444 .000 nuevos pesos (de esa época), de los cuales hubo un aporte del $36 \%$ del Banco Mundial, un $13.9 \%$ de recursos crediticios, el $26 \%$ del programa nacional de solidaridad y el $24 \%$ por parte del Ayuntamiento. La infraestructura consistía en un camino de acceso con pavimentación, sistema de control de lixiviados, pozos de monitoreo captadores de biogás, impermeabilización, maquinaria y equipo para el manejo de la basura, además de que se encontraba complementada de un estudio de factibilidad para solventar los créditos obtenidos para su construcción (AyunTAmiento DE Othón P. Blanco, s. f. d).

Para el período de gobierno municipal de 1996-1999, se trabajaba diariamente con 290 personas, quienes se dedicaban al barrido, pepenado y recolección de basura, chapeo y podado de camellones, parques, jardines y otros. La producción de basura alcanzaba las 301 toneladas/diarias (t/d), además de que se atendía la totalidad de las colonias en Chetumal y Calderitas (Ayuntamiento de Othón P. Blanco, s. f. e).

\subsection{Políticas públicas para la gestión sostenible del turismo}

La década de los años dos mil se caracterizó por esfuerzos para controlar tiraderos a cielo abierto y por la colaboración de organismos internacionales, como la Agencia de Cooperación 


\section{El manejo de los residuos sólidos y la actividad turística \\ en Chetumal, México: una relación compleja}

Internacional de Japón y la Agencia Alemana de Cooperación Técnica, que sumaron esfuerzos para el diagnóstico situacional en materia de RS. Posteriormente se llevó a cabo el saneamiento del sitio del relleno sanitario, el fortalecimiento de la recolección y la implementación de programas de educación ambiental a la ciudadanía y a población escolar través de la Secretaría de Educación y Cultura en Quintana Roo (Ayuntamiento de Othón P. Blanco, 2003).

En la administración municipal de los años 2005-2008, se presentaron diversas innovaciones relacionadas con la educación ambiental a la ciudadanía a través del programa llamado "Municipio Limpio". Este programa hacía énfasis en la minimización de la generación y el manejo adecuado de los RS. Además, se completaba con la motivación al ciudadano para el mantenimiento de patios, frentes, calles y lugares públicos limpios; asimismo, la inclusión de instituciones estatales y nacionales (Ayuntamiento de Othón P. Blanco, 2006).

En esa administración se gestionó la adquisición de ocho camiones para el fortalecimiento del servicio de recolección, con lo que se alcanzó el 100 \% de recolección en las 91 colonias hasta esas fechas, recolectándose entre 190 y 200 t/d de RS (Ayuntamiento de Othón P. Blanco, 2007). Además, se trabajó en actividades como la descacharrización de patios, lotes baldíos e inclusive el saneamiento de zonas costeras, como la bahía de Chetumal (AyunTAmiento DE Отнón P. BLANCo, 2008).

En el año 2010 se recolectaban en Quintana Roo un total de 1644 t/d de RS de viviendas, parques, jardines y edificios públicos; mientras que en el municipio de Othón P. Blanco se recolectan $300 \mathrm{t} / \mathrm{d}$, y ya se contaba con veintitrés vehículos recolectores, más el actual relleno sanitario como medidas técnicas de GRS (INEGI, 2014). Sin embargo, la percepción ante el servicio (manejo de RS) se calificaba como deficiente por parte de la ciudadanía:

(El servicio) no es muy bueno porque he observado colonias en donde la basura permanece tirada en una esquina por semanas sin que el camión la recolecte (mujer, ama de casa 66 años, comunicación personal, diciembre de 2015).

Este descontento en la percepción se relacionaba principalmente con la frecuencia de recolección en las colonias, aunado a la falta de programas de concienciación ambiental a la población:

Debería haber más programas para concientizar a la gente y multar al que tire basura en la calle. Nuestra ciudad no es limpia. La mayoría de la gente es inconsciente y botan la basura en todos lados (mujer, ama de casa, 48 años, comunicación personal, diciembre de 2015). 
A pesar de que se señalaba al gobierno como principal responsable, también se aludía a una conciencia ciudadana de involucramiento en la GRS:

Creo que es parte de la obligación del gobierno resolver los problemas que genere la basura, también creo que la población está obligada a mejorar la clasificación de la basura, si hay personas que pueden obtener beneficios económicos o alimenticios por la basura reciclable, pues está bien (mujer, burócrata, 33 años, comunicación personal, diciembre 2015).

Otra de las problemáticas denunciadas por los ciudadanos hacía referencia a la separación en el origen y el reciclaje, que si bien podría representar una oportunidad de intercambio económico en beneficios económicos a las familias, reconocieron que esta práctica tiene que estar liderada por el gobierno, lo cual debería reflejarse en campañas de separación y reajuste técnico de la recolección:

En cuanto a la separación de residuos, aunque en casa los separamos, al pasar el camión de la basura, todo va a parar al mismo lugar. A mi manera de ver las cosas, eso no tiene sentido; yo cumplo con separar (mujer, profesionista, 54 años, comunicación personal, diciembre de 2015).

Como parte de las estrategias institucionales del gobierno estatal (2011-2016), desde 2012 y hasta finales de 2016, se implementó el programa "Reciclando Basura por Alimentos" (RBA), el cual se regía por el discurso político y mediático del fomento al reciclaje (Grupo FórmuLA, 2014). La estrategia del programa fue el acopio de RS reciclables, como cartón, vidrio, plástico y aluminio, que eran intercambiados a la ciudadanía por productos como arroz, aceite, frijol, jabón, productos enlatados, productos de limpieza y de aseo personal, harina, lentejas, galletas, pan, entre otros (GaLICIA, 2017; Blanco, 2018) (ver figura 5).

Figura 5. Programas de participación ciudadana para la gestión de residuos sólidos y el reciclaje en Chetumal
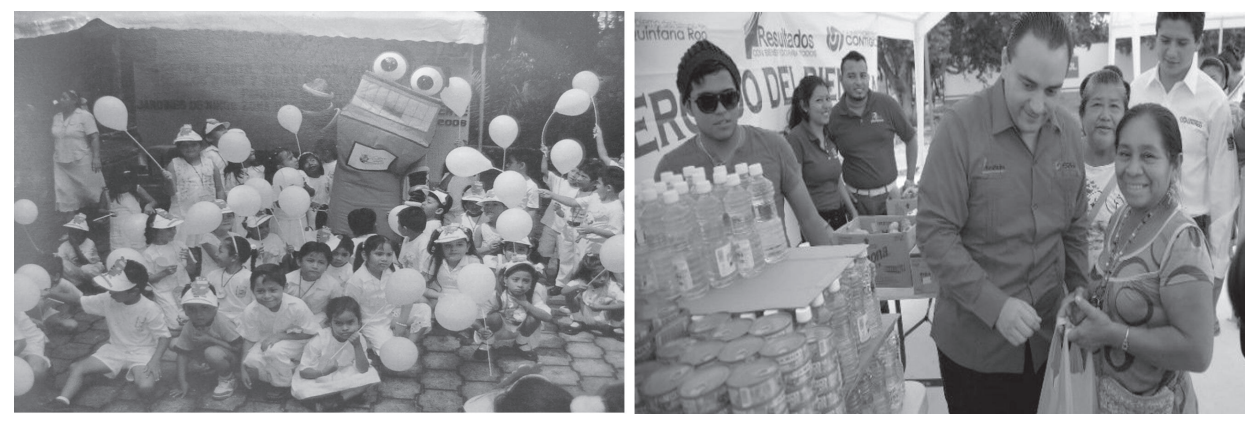


\section{El manejo de los residuos sólidos y la actividad turística en Chetumal, México: una relación compleja}

La naturaleza de programas como RBA ha demostrado fortalecer la participación de los ciudadanos a través de incentivos. Como casos de éxito la literatura reporta a Curitiba, Brasil, donde se intercambiaron vales de transporte público por RS, o el caso mexicano en Cuauhtémoc, México, en el cual, por cada seis bolsas de RS se intercambiaban productos de la canasta básica (Programa Ambiental de Naciones Unidas; cp. Hettiarachchi, Ryu, Caucci y Silva (2018).

El caso de RBA se oscurece por la mínima participación de la ciudadanía, pues los registros muestran una participación de tan solo el 1 \% del total de familias de Chetumal (León-López, BojórQuez y GonzÁlez (2018). Este porcentaje también coincide con la opinión pública al calificarlo como un programa paliativo a la situación de los RS en la ciudad (LeóN-LóPez, 2016):

(RBA) es un paliativo temporal, no resuelve el problema de fondo, se requiere educación y programas gubernamentales coherentes, así como suficientes camiones recolectores, construcción de un verdadero relleno sanitario, sueldos decorosos para que las personas no mendiguen alimentos (mujer, burócrata, 50 años, comunicación personal, diciembre de 2015).

Mientras tanto, en el contexto general de la ciudad, se observaba una afluencia turística al alza (figura 6). Tan solo en el año 2017, se registraron cerca de 495.976 visitantes (SEDETUR, 2018), lo cual es comparable con destinos consolidados como la ciudad de San Miguel de Allende en Guanajuato, que en ese mismo año registró 556.799 llegadas de turistas (SECRETAría de Turismo del Estado de Guanajuato, 2017).

En las gráficas de los visitantes para Chetumal (figura 7) se muestra que, a pesar de la afluencia registrada para la ciudad, denota una tendencia a la baja para lo correspondiente al caso México-Belice (SEDETUR, 2018), que no necesariamente refleja la situación actual, ya que es sabido que esta afluencia se muestra al alza en diarios locales ${ }^{3}$, los cuales han reportado la espera de hasta de 58.000 visitantes beliceños tan solo en vacaciones de verano, con un gasto de $\$ 600,00$ (seiscientos pesos mexicanos M/N) por visitante (TejEDA, 2019), por lo que estos datos pueden prestarse a inconsistencias y confusión.

El éxito en el indicador turístico de Chetumal invita a una reflexión más con respecto al impacto ambiental y la generación de RS, ya que a mayor afluencia de visitantes se espera una mayor cantidad en la producción con respecto a los generados en la vida cotidiana de los anfitriones. Sin embargo, al comparar con destinos como San Miguel de Allende, se encuentra que, a pesar de que en la ciudad colonial se recibe mayor afluencia de visitantes, se genera menor cantidad de RS que en Chetumal $^{4}$ (INEGI, 2014). Esto podría estar relacionado con el
3. A nivel cualitativo, los diarios locales han difundido titulares como "Aumenta flujo de personas que cruzan de Belice a México" (CASTILLA, 2018) o "Empresarios 'se frotan las manos' por turismo beliceño" (TEJEDA, 2019), lo cual denota aumento de turistas beliceños por razones de placer y compras en comercios locales.

4. De acuerdo con el INEGI (2010), San Miguel de Allende como municipio contó con 160.383 habitantes, mientras que el municipio de Othón P. Blanco, del cual Chetumal es cabecera municipal, contó con 154.243 habitantes. Sin embargo, en San Miguel se generaron $90 \mathrm{t} / \mathrm{d}$ de $R S$, mientras que en Chetumal, 300 t/d (INEGI, 2014). 
tipo de turista, los hábitos de la población anfitriona e inclusive el interés gubernamental en la temática (Richie y Crouch, 2003; Paramati, Shahbaz y Alam, 2017).
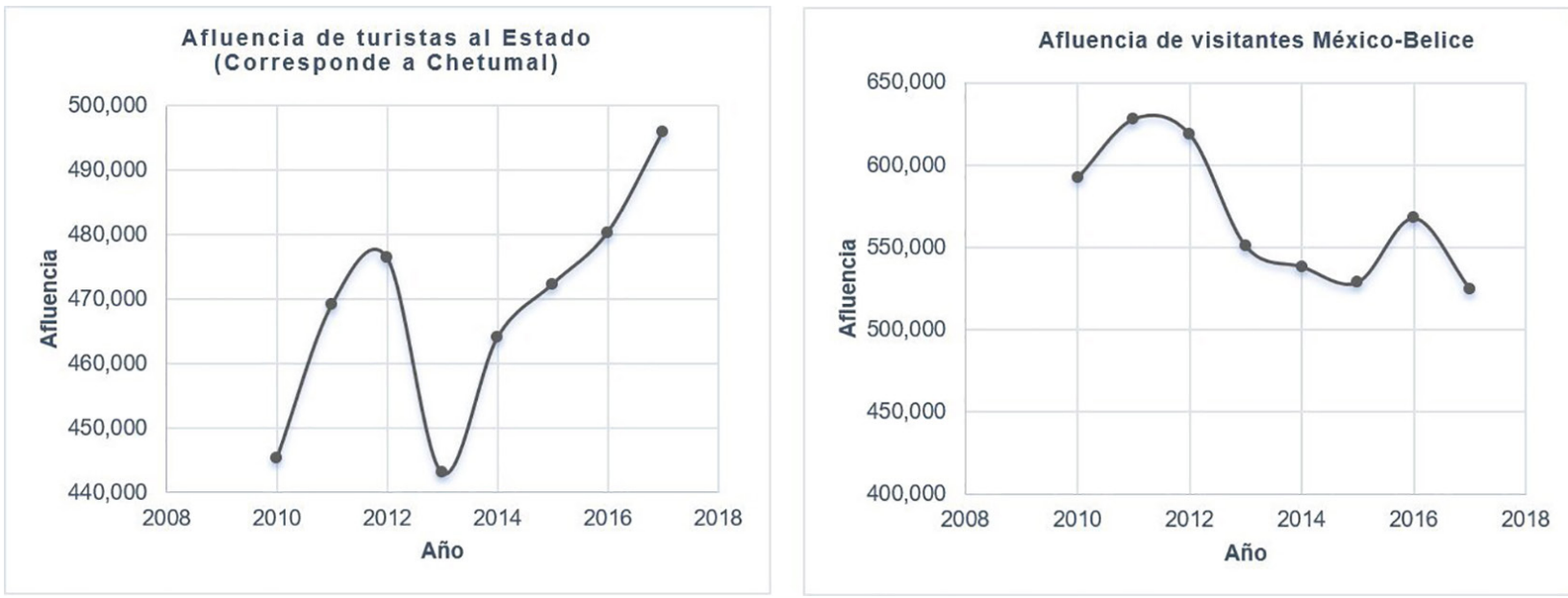

Figura 6. Tendencias en la afluencia de turistas a Chetumal (izquierda) y visitantes México-Belice (derecha)

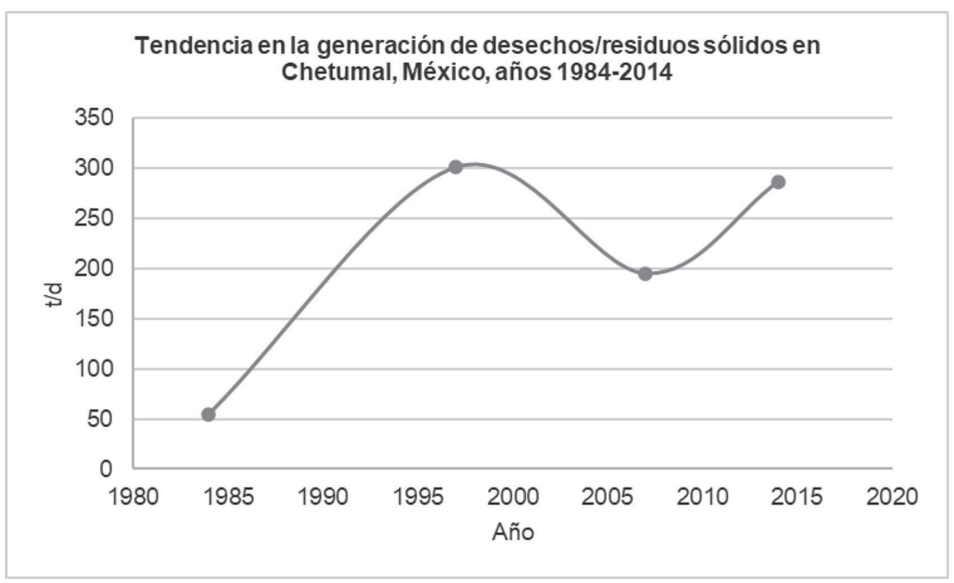

Figura 7. Tendencia en la generación de desechos/residuos sólidos en la ciudad de Chetumal, México 


\section{Acerca de la interacción entre los elementos} de la gestión: escala estructural-funcional

\subsection{Aspectos externos (contexto turístico-fronterizo)}

El contexto turístico y fronterizo de la ciudad dicta la necesidad de encontrar formas de cooperación internacional para la sostenibilidad ambiental de la región fronteriza Belice-México. Esto se vincula estrechamente con el fortalecimiento del sistema jurídico y administrativo en ambos países, para que se tome en cuenta la presión que ejerce el turismo binacional.

Con respecto al reto en la comprensión de la apropiación del espacio turístico por parte de la población beliceña, se abre la brecha para una política internacional de concientización ambiental con relación a la importancia turística de todos los sectores sociales involucrados - como pueden ser el sector hotelero, transporte y centros comerciales-. Debido a que la problemática se encuentra vinculada con los comportamientos de los turistas y el estilo normativo de cómo deben ser gestionados los RS a nivel local, es pertinente encontrar mecanismos para el cobro consciente de impuestos y demás instrumentos jurídicos que velen por la sostenibilidad ambiental (BudEANu, 2007). Sobre esa base, habrá que fortalecer los levantamientos estadísticos binacionales para una mejor instrumentación de planes y estrategias ambientalmente sostenibles, aunado al fomento de mayor participación, cohesión e inclusión de la sociedad civil y empresarial.

\subsection{Elementos para el flujo de los RS}

Los RS generados en Chetumal tienen origen tanto en las actividades cotidianas como en las de recreación y turísticas. Si bien los principales generadores son las casas-habitación por el número de habitantes, la acumulación es llevada a cabo en espacios comunes para la recreación y el turismo, siendo estos áreas consideradas como parte de la oferta turística (Molina, 2005).

Tradicionalmente, el estilo de mitigación de dicha acumulación de los RS generados en la ciudad ha sido el barrido mecánico de parques, avenidas y demás oferta turística, así como la recolección puerta a puerta en las casas-habitación, con fuerte peso en la mano de obra de los trabajadores municipales encargados, e inclusive la participación de la ciudadanía por mantener su frente domiciliario limpio, lo cual devela la falta de una política de educación y concienciación ambiental eficaz a nivel poblacional. 
Para efectos de la disposición final, se recomienda la clausura y saneamiento del sitio que, a pesar de contar con la infraestructura para operar como relleno sanitario, se desempeña como tiradero a cielo abierto que afecta a colonias aledañas. Por lo tanto, los tomadores de decisión locales podrían revisar experiencias exitosas de la coordinación en asociaciones público-privadas y de las ONG, para la resolución a esquemas de mayor participación social, preservación ambiental y beneficios económicos (HetTiarachchI et ÁL., 2018).

\subsection{El papel de los agentes sociales involucrados}

Para Chetumal, la realidad en el contexto turístico ha promovido la necesaria inclusión de la participación ciudadana en programas de descacharrización y campañas de educación ambiental. Sin embargo, a pesar del interés gubernamental en este rubro, ha habido dificultades para generar un sentido de compromiso en la ciudadanía, asimismo en los rubros empresariales.

De 2010 a la fecha no se registró un avance significativo en la ejecución de políticas públicas de gestión para un manejo adecuado. Por otro lado, la ejecución de programas como el llamado RBA se vio oscurecida por su alta mercadotecnia política, lo cual limitó su permeabilización a toda la población (LEÓN-LóPEZ ET AL., 2018). La debilidad principal en la ejecución de este tipo de programas reside en no proporcionar la información necesaria al ciudadano acerca de la importancia de la separación de los RS y del reciclaje (Valencia, Espinosa, ParRA Y PeÑA, 2011).

Se devela que adherir la GRS al contexto económico-turístico-fronterizo permitiría la visualización como un problema de desarrollo regional en el que se podrían sumar las firmas de la oferta turística en conjunto con los agentes sociales nativos (gobierno y ciudadanía) del sistema actual, debido a que el objetivo común podría ser posicionar e incrementar la competitividad ambiental del DT. El principal beneficio esperado sería un mejor desempeño económico e incremento de la competitividad ambiental, debido a que se gestaría una forma de gobernanza funcional en la cooperación estratégica de políticas públicas ad hoc a la problemática, y optimizar la experiencia del visitante (NAVA y VaLenzuela, 2014).

\section{Conclusión}

La importancia de analizar la problemática de los RS en DT facilita no solo encaminar su resolución, sino que la incluye oficialmente en la agenda de los impactos del turismo. Esto 
ayuda a develar futuros escenarios sostenibles para los sectores sociales, empresariales y gubernamentales, para que juntos encaminen la gestión integral con dirección hacia una política de generadores responsables. En Chetumal se deberá seguir con mecanismos de concientización y educación ambiental a través de políticas inclusivas de participación ciudadana, voluntad política y planteamientos comunes junto al sector empresarial, en beneficio de la competitividad ambiental del DT, lo cual ayudaría a ampliar el espectro de acción política para el manejo integral a través de una nueva cultura de gobernanza.

El enfoque de complejidad permite adscribir la GRS en su entorno contextual de DT y zona fronteriza, que, haciendo énfasis en las escalas espacio-temporales, devela las relaciones entre los agentes sociales y la manera como han interactuado en la evolución del sistema. Por otro lado, este abordaje podría promover futuras investigaciones en las que se estudie la embrionaria estructura de gobernanza en la ciudad en cuestión y los mecanismos necesarios para la transición hacia la sostenibilidad de la ciudad y la conservación de su patrimonio natural.

\section{Agradecimientos}

Parte de esta investigación fue realizada bajo el financiamiento del CONACYT mediante la beca de posgrado con número 277362 otorgada al primer autor; asimismo se agradece al Mtro. Romeo Alejandro Sánchez Zavalegui por el apoyo técnico en la elaboración del mapa de ubicación de Chetumal.

\section{Referencias bibliográficas}

ABARCA-MORENO, Liliana; MAAS, Ger y HOGLAND, William (2015). Desafíos en la gestión de residuos sólidos para las ciudades de países en desarrollo. Tecnología en Marcha, 28(2), pp. 141-168. https://doi.org/10.18845/tm.v28i2.2340

AYUNTAMIENTO DE OTHÓN P. BLANCO (s. f. a). Primer Informe de Gobierno Municipal del H. Ayuntamiento de Othón P. Blanco Q. Roo 1981-1984. Chetumal, México: H. Ayuntamiento de Othón P. Blanco, 32 pp.

AYUNTAMIENTO DE OTHÓN P. BLANCO (s. f. b). Tercer Informe de Gobierno Municipal del H. Ayuntamiento de Othón P. Blanco Q. Roo 1981-1984. Chetumal, México: H. Ayuntamiento de Othón P. Blanco, 55 pp. 
AYUNTAMIENTO DE OTHÓN P. BLANCO (1991). Primer informe de gobierno 1990-1993. Chetumal, México: Ayuntamiento de Othón P. Blanco, 35 pp.

AYUNTAMIENTO DE OTHÓN P. BLANCO (s. f. c). II Informe de Gobierno Municipal. Chetumal, México: Ayuntamiento Othón P. Blanco 1990-1993, 61 pp.

AYUNTAMIENTO DE OTHÓN P. BLANCO (s. f. d). III Informe de Gobierno Municipal. Chetumal, México: Ayuntamiento Othón P. Blanco 1990-1993, 40 pp.

AYUNTAMIENTO DE OTHÓN P. BLANCO (s. f. e). Primer Informe de Gobierno Municipal. Chetumal, México: Editora Norte Sur, $40 \mathrm{pp}$.

AYUNTAMIENTO DE OTHÓN P. BLANCO (2003). Primer Informe de Gobierno. Cancún, México: Grupo Editorial Regiomontano, $68 \mathrm{pp}$.

AYUNTAMIENTO DE OTHÓN P. BLANCO (2006). Primer Informe de Gobierno Municipal. Chetumal, México: Alfa/Zeta, 148 pp.

AYUNTAMIENTO DE OTHÓN P. BLANCO (2007). Segundo Informe de Gobierno Municipal. Chetumal, México: Alfa/Zeta, $262 \mathrm{pp}$.

AYUNTAMIENTO DE OTHÓN P. BLANCO (2008). Tercer Informe de Gobierno Municipal. Chetumal, México: Alfa/Zeta, 298 pp.

BAGGIO, Rodolfo; SCOTT, Noel \& COOPER, Chris (2010). Improving tourism destination governance: a complexity science approach. Tourism Review, 65(4), 51-60. http://dx.doi.org/10.1108/16605371011093863

BENI, Mario (1998). Análise estrutural do turismo. São Paulo: SENAC.

BLANCO, Stephani (24 de junio de 2018). Borge recibió millonario recurso por Basura por alimentos. Novedades de Quintana Roo. Recuperado de https:// sipse.com/novedades/borge-recibio-millonario-recurso-por-basura-poralimentos-301530.html

BUDEANU, Adriana (2007). Ustainable tourist behaviour a discussion of opportunities for change. International Journal of Consumer Studies, 31(2007), pp. 499-508.

BUSTOS, Carlos y CHAcón, Galia (2009). El desarrollo sostenible y la agenda 21. Telos, 11(2), pp. 164-181. Recuperado de https://www.redalyc.org/ pdf/993/99312517003.pdf

CASTILLA, Ángel (12 de agosto de 2018). Cónsul recomienda mejor trato a turistas beliceños. Novedades de Quintana Roo. Recuperado de https://sipse. com/novedades/chetumal-turismo-beliceno-afluencia-semana-santaatencion-trato-visitantes-compras-belicenos-derrama-economica-capitalciudad-292025.html 
ECOS DEL CARIBE (julio de 1976). Nuevos métodos de recolección de basura. Ecos del Caribe, 3 y 5 pp.

GALICIA, Alejandra (7 de febrero 2017). Hallan anomalías en Basura por Alimentos. Novedades de Quintana Roo. Recuperado de https://sipse.com/novedades/ hallan-anomalias-en-basura-por-alimentos-programa-recursos-robertoborge-recursos-cancun-241772.html

GAMBOA, Liliana y CARRILLO, Citlali (2016). Contaminación por residuos sólidos en la bahía de Chetumal, Quintana Roo. En Encuentro de Expertos en Residuos Sólidos, Los Residuos Sólidos como fuente de materiales y energía. México: Sociedad Mexicana de Ciencia y Tecnología Aplicada a Residuos Sólidos A.C., pp. 282-287. Recuperado de: http://www.somers-ac.org/encuentros/ encuentros_resource/Encuentro9.pdf

GARCÍA, Densis y OROPEZA, Norma (2015). Determinación de microplásticos en arenas de la bahía de Chetumal, Quintana Roo, México. En VI Simposio Iberoamericano en Ingeniería de Residuos Sólidos, Hacia la Carbono Neutralidad 2021. Costa Rica: Editorial Tecnológica de Costa Rica, pp. 675676. Recuperado de: https://repositoriotec.tec.ac.cr/handle/2238/9221

GEISSDOERFER, Martin; SAVAGET, Paulo; BOCKEN, Nancy \& HULTINK, Erik (2016). The Circular Economy - A new sustainability paradigm? Journal of Cleaner Production, 143. 757-768. http://dx.doi.org/10.1016/j.jclepro.2016.12.048

GERSHENSON, Carlos (2013). ¿Cómo hablar de complejidad? Llengua, Societat i Comunicació. https://doi.org/10.1344/LSC-2013.11.3

GOBIERNO DEL TERRITORIO DE QUINTANA ROO (1967). Informe de actividades. Chetumal: Talleres Gráficos del Gobierno del Estado de Quintana Roo.

GONZÁLEZ, Alfonso y MACÍAS, Alma (2010). Tres décadas como anfitriona: génesis, expansión y crisis de la sociedad quintanarroense. Revista de Economía, Sociedad, Turismo y Medio Ambiente, (11), pp. 191-192.

GRUPO FÓRMULA (15 de abril de 2014). Reciclando Basura por Alimentos mejor imagen de colonias de Quintana Roo. Radio Fórmula. Recuperado de http://www. radioformula.com.mx/notas.asp? Idn $=404995 \&$ idFC $=2014$

GUEVARA, José y FLORES, Patricia (2011). Caracterización de residuos sólidos domiciliarios en Quintana Roo. En Sara Ojeda; Samantha Cruz; Paul Taboada y Quetzalli Aguilar (coords.), Hacia la sustentabilidad: los residuos sólidos como fuente de materia prima y energía. México: Universidad 
Autónoma de Baja California, pp. 31-35. Recuperado de: http://www. somers-ac.org/encuentros/encuentros_resource/Encuentro4.pdf

GUEVARA, José; FLORES, Patricia; CANUL, Suhail; ABURTO, David, y ROMERO, Geovanny (2011). "Caracterización y cuantificación de subproductos de residuos sólidos en las playas del sur de la Costa Maya, Quintana Roo”. En Sara Ojeda; Samantha Cruz; Paul Taboada y Quetzalli Aguilar (coords.), Hacia la sustentabilidad: los residuos sólidos como fuente de materia prima y energía. México: Universidad Autónoma de Baja California, pp. 6063. Recuperado de: http://www.somers-ac.org/encuentros/encuentros_ resource/Encuentro4.pdf

GUILARDUCCI, Bruno \& FRATUCCI, Aguinaldo (2020). Analysis of the social network of the Instance of Governance of the Caminho Novo Tourist Circuit, MG: a complex and systemic perspective. Revista Brasileira de Pesquisa em Turismo, 14(1), pp.140-160. DOI: 10.7784/rbtur.v14i1.1734

HETTIARACHCHI, Hiroshan; RYU, Sohyeon; CAUCCI, Serena, y SILVA, Rodolfo (2018). Municipal Solid Waste Management in Latin America and the Caribbean: Issues and Potential Solutions from the Governance Perspective. Recycling, 3(19). Recuperado de http://collections.unu.edu/eserv/UNU:6506/ recycling-03-00019-1.pdf

INEGI (2010). Censo de población y vivienda 2010. Principales resultados por localidad. Recuperado de: http://www.inegi.org.mx/sistemas/consulta_resultados/ iter2010.aspx

INEGI (2014). Residuos sólidos. Recuperado de http://www.beta.inegi.org.mx/temas/residuos/ JACOMY, Bruno (1992). Historia de las técnicas. Buenos Aires: Losada.

JIMÉNEZ, Nancy (2015). La gestión integral de residuos sólidos urbanos en México: entre la intención y la realidad. Letras Verdes. Revista Latinoamericana de Estudios Socioambientales, (17), pp. 29-56. doi:10.17141/letrasverdes.17.2015.1419

JIMÉNEZ, Nancy (2017). El residuo: producto urbano, asunto de intervención pública y objeto de la gestión integral. Culturay representaciones sociales, 11(22), pp. 158-192. Recuperado de: http://www.scielo.org.mx/scielo.php?script=sci_ arttext\&pid=S2007-81102017000100158

KEN, Crucita (2014). Desarrollo Regional y Gestión Pública en Quintana Roo, 1970 a 2010: Estudio de caso, municipio de Othón P. Blanco. México: Editorial Porrúa.

LGPGIR. Diario Oficial de la Federación, Ciudad de México, 19 de enero de 2018. 
LEÓN-LÓPEZ, Armando (2016). Potencialidades en la gestión y el manejo para la valorización social de los Residuos Sólidos Urbanos en Chetumal, Quintana Roo (tesis de maestría en planeación). Universidad de Quintana Roo, Chetumal, México.

LEÓN-LÓPEZ, Armando; BOJÓRQUEZ, Inocente y GONZÁLEZ, Alfonso (2018). Evaluación de un Programa de Fomento al Reciclaje en el Caribe Mexicano. En Miguel Barrera (comp.), Diseño, Implementación y Evaluación de Políticas Públicas. Estudios de caso en México y América Latina, Chetumal, México: Universidad de Quintana Roo, pp. 323-338.

MACÍAS, Gabriel (2004). Estudio introductorio. En Gabriel Macías (Coord.), El vacío imaginario. Geopolítica de la ocupación territorial en el Caribe oriental mexicano. México: Centro de Investigaciones y de Estudios Superiores en Antropología Social y H. Congreso del Estado de Quintana Roo.

MANDUJANO, Porfirio (2002). Análisis del impacto ambiental del relleno sanitario sobre la bahía de Chetumal. En Francisco Rosado; Rafael, Romero y Alberto Navarrete (eds.), Contribuciones de la ciencia al manejo costero integrado de la Bahía de Chetumal y su área de influencia. México: Universidad de Quintana Roo, pp. 211-218.

MOLINA, Sergio (2005). Conceptualización del turismo. México: Limusa.

NAVA, Miriam y VALENZUELA, Blas (2014). Acción colectiva y gobernanza del Centro Histórico de Mazatlán, México. Recuperación y conservación a espacio turístico. Ánfora, 21(36), pp. 125-148.

NOVEDADES DE QUINTANA ROO (22 de enero de 1975). Previene Turismo la necesidad de una Campaña de Limpieza. Novedades de Quintana Roo, 1 y 5 pp.

ORGAZ, Francisco y MORAL, Salvador (2016). El turismo como motor potencial para el Desarrollo económico de zonas fronterizas en vías de Desarrollo. Un estudio de caso. El Periplo Sustentable, 30. Recuperado de: http://www.redalyc. org/articulo.oa?id=193449985009

PODER EJECUTIVo (2010). Constitución Política de los Estados Unidos Mexicanos. México.

PARAMATI, Sudharshan; SHAHBAZ, Muhammad, y ALAM, Samsul (2017). Does tourism degrade environmental quality? A comparative study of Eastern and Western European Union. Transportation Research Part D: Transport and Environment, 50, pp. 1-13. https://doi.org/10.1016/j.trd.2016.10.034

PULIDO-FERNÁNDEZ, Juan y PULIDO-FERNÁNDEZ, María (2015). ¿Sigue vigente el paradigma del turismo sostenible? Reflexiones a la luz de la literatura reciente. 
PASOS. Revista de Turismo y Patrimonio Cultural, 13(6). Recuperado de: http://www.redalyc.org/articulo.oa?id=88143407004

QUEQUI (3 de marzo de 2017). Desvió Borge 67 MDP de Basura por Alimentos para gasto electoral. Quequi. Recuperado de https://www.quequi.com.mx/desvioborge-67-mdp-de-basura-por-alimentos-para-gasto-electoral/

RITCHIE, Brent y CROUCH, Geoffrey (2003). The competitive destination: A sustainable tourism perspective. Melbourne, Australia: CABI publishing.

SECRETARÍA DE AGRICULTURA Y FOMENTO (1918). Informe de la Comisión Geográfico-Exploradora de Quintana Roo. México: Oficina impresora de la Secretaría de Hacienda, Departamento de Fomento.

SECRETARÍA DE TURISMO DEL ESTADO DE GUANAJUATO (2017). Actividad turística del estado de Guanajuato. Boletín mensual. Dirección General de Planeación. Secretaría de Turismo del Estado de Guanajuato. Recuperado de http:// www.observatorioturistico.org/cenDoc/a59f3-Bolet--n-Act.-Tur--sticaDiciembre-2017.pdf

SECTUR (2018). Datatur3 -Visitantes Internacionales. Secretaría de Turismo Recuperado de https://www.datatur.sectur.gob.mx/SitePages/VisitantesInternacionales.aspx

SEDETUR (2018). Indicadores Turísticos. Secretaría de Turismo del Estado de Quintana Roo. Recuperado de http://sedetur.qroo.gob.mx/index.php/estadisticas/ indicadores-turisticos

TEJEDA, Enrique (2 de julio 2019). Empresarios 'se frotan las manos' por turismo beliceño. Novedades Quintana Roo. Recuperado de https://sipse.com/novedades/esperan-millonaria-derrama-chetumal-temporada-vacacional-turismo-derrama-belice-337408.html

VALENCIA, Jesús; ESPINOSA, Adriana; PARRA, Adela, y PEÑA, Miguel (2011). "Percepción del riesgo por emisiones atmosféricas provenientes de la disposición final de residuos sólidos”. Revista de Salud Pública, 13(6), pp.930-941. http://www.redalyc.org/articulo.oa?id=42222537006

VARGAS, Mario (Coord.). (2014). Antología de las legislaturas de Quintana Roo, 1974-2014. México: H. Congreso del Estado de Quintana Roo, 230 pp.

VIGURI, Miguel (2019). Ciencias de la complejidad vs. pensamiento complejo. Claves para una lectura crítica del concepto de cientificidad en Carlos Reynoso. PENSAMIENTO, 75(283), 87-106. doi: 10.14422/pen.v75.i283.y2019.004 